\title{
Viabilidade econômica de sistemas agroflorestais em Novo Progresso (PA)
}

Os SAFs são o conjunto de técnicas de manejo e uso da terra que implicam na combinação de árvores com cultivos perenes, com pecuária ou com ambas as proposições, de forma conjunta ou escalonada no tempo e no espaço. 0 objetivo deste trabalho foi realizar uma estimativa de geração de renda, por meio de uma análise de viabilidade econômica, pela introdução de sistemas agroflorestais (SAFs), possibilitando ao produtor a tomada de decisões em seu investimento antes mesmo de este ser implementado para minimizar frustrações e perda de capital em propriedades de agricultores familiares no Projeto de Desenvolvimento Sustentável Terra Nossa, localizado nos municípios de Novo Progresso e Altamira. Ao todo, 44 unidades familiares distribuídas aleatoriamente no assentamento demonstraram interesse em participar da implantação de SAF promovidos pelo Projeto Horizonte Verde. Foram selecionados 18 produtores, distribuídas aleatoriamente no assentamento demonstraram interesse em participar da implantação de SAF promovidos pelo Projeto Horizonte Verde. Foram selecionados 18 produtores, municipal de Novo Progresso, por meio de aplicação de questionário, para fins de constatação dos valores de produtos como sementes, frutos, madeira, cereais entre outros produtos. Verificou-se que, com exceção do arranjo S4, grande parte dos arranjos de SAFs analisados mostrou ter uma estimativa de renda positiva, pois os valores para Valor Presente Líquido (VPL), taxa interna de retorno e relação custo-benefício foram positivos em um horizonte de 20 anos. Com relação às receitas e saldos nos SAFs, observa-se que os seis arranjos mais rentáveis, classificados pelo critério do VPL, foram os arranjos $\mathrm{S} 18, \mathrm{~S} 2, \mathrm{~S} 10, \mathrm{~S} 11$, S1 e S12 que se destacaram por ter espécies com alta produtividade anual e aceitação no mercado local. A maioria do SAFs analisados aponta viabilidade econômica de acordo com os indicadores de VPL, TIR e RB/C, possibilitando ao produtor tomar decisões de investimento. Os SAFs mais rentáveis economicamente apresentaram em sua composição açaí (Euterpe oleracea Mart.) e cumaru (Dipteryx odorata Willd). Os valores gastos com tratos culturais e colheita, são os que mais demandam mão de obra, sendo responsáveis por boa parte dos custos totais dos SAFs na estimativa realizada para 20 anos.

Palavras-chave: Rendimento agroflorestal; Agricultura familiar; Análise econômica.

\section{Economic viability of agroforestry systems in Novo Progresso (PA)}

SAFs are the set of management and land use techniques that involve the combination of trees with perennial crops, with livestock or with both propositions, together or staggered in time and space. The objective of this work was to estimate income generation through an economic viability analysis, by introducing agroforestry systems (SAFs), allowing producers to make decisions on their investment even before it is implemented to minimize frustrations. and loss of capital on family farmers' properties in the Terra Nossa Sustainable Development Project, located in the municipalities of Novo Progresso and Altamira. In all, 44 family units randomly distributed in the settlement showed interest in participating in the implementation of AFS promoted by the Green Horizon Project. Eighteen producers were selected, and questionnaires were applied in the form of personal interviews. To compose the value of the revenues, data were obtained on the market prices of the products sold at the Novo Progresso municipal headquarters, through the application of a questionnaire to verify the values of products such as seeds, fruits, wood, cereals among other products. With the exception of the S4 arrangement, most of the analyzed SAF arrangements were found to have a positive income estimate, since the values for Net Present Value (NPV), internal rate of return and cost-benefit ratio were positive in one. 20-year horizon. With regard to SAF revenues and balances, it is observed that the six most profitt a most proft (Dins. The most the most labor intensive, accounting for much of the total costs of SAFs in the estimate made for 20 years.

Keywords: Agroforestry yield; Family farming; Economic analysis.

Topic: Ciências Florestais

Reviewed anonymously in the process of blind peer.
Received: 02/07/2018

Approved: $15 / 07 / 2018$
Saulo Ubiratan Pinheiro da Silva (iD)

Universidade Federal do Oeste do Pará, Brasil

http://lattes.cnpq.br/7489857515370911

http://orcid.org/0000-0002-3114-5814

saulostm@gmail.com

Daniela Pauletto (iD)

Universidade Federal do Oeste do Pará, Brasil http://lattes.cnpq.br/0963317170667125

http://orcid.org/0000-0003-1855-6077

danielapauletto@hotmail.com

Cléo Gomes da Mota (iD)

Universidade Federal do Oeste do Pará, Brasil

http://lattes.cnpq.br/9028347821830300

http://orcid.org/0000-0002-7689-6718

motacleo@hotmail.com
Gabriela de Cássia Santos do Nascimento

Universidade Federal do Oeste do Pará, Brasil http://lattes.cnpq.br/6334547766236039

gabriela.cassia1@gmail.com

Juliana Andressa Costa dos Santos

Universidade Federal do Oeste do Pará, Brasil

http://lattes.cnpq.br/9872151304772567

julianaandressa.c.s@gmail.com
Referencing this:

SILVA, S. U. P.; PAULETTO, D.; MOTA, C. G.; NASCIMENTO, G. C. S.; SANTOS, J. A. C.; RODE, R.; NOCE, R.. Viabilidade econômica de sistemas agroflorestais em Novo Progresso (PA). Revista Ibero Americana de Ciências Ambientais, v.9, n.6, p.28-36, 2018. DOI: http://doi.org/10.6008/CBPC2179-6858.2018.006.0003 


\section{INTRODUÇÃO}

Grande parte das áreas degradadas do país encontram-se na região amazônica, tendo em vista seus modelos históricos de desenvolvimento e ocupação que não contemplaram aspectos ambientais (INPE, 2007). O desmatamento na Amazônia registrou em 2004 uma média de $27.772 \mathrm{~km}^{2}$, o segundo maior registro do bioma. A taxa de desmatamento manteve-se estagnada entre 2009 e 2015 em um patamar médio de $6.080 \mathrm{~km}^{2}$ por ano. No entanto as taxas registradas pelo INPE aumentaram, chegando a $2016 \mathrm{em} 7.989 \mathrm{~km}^{2}$, o maior registro desde 2008 (AZEVEDO, 2016).

O Governo Federal criou iniciativas que visam recuperar áreas degradadas, dentre as quais o Plano de Agricultura de Baixa Emissão de Carbono (Plano ABC), criado em 2012, que tem como objetivo principal reduzir a taxa de desmatamento na Amazônia em $80 \%$ e $40 \%$ no Cerrado. Uma das formas do Plano ABC atingir seu objetivo é por meio da recuperação de áreas degradadas com implantação de Sistemas Agroflorestais (SAFs).

Considerando esta tendência de redução e combate ao desmatamento, o Projeto Horizonte Verde, desenvolvido pela Organização Não Governamental Instituto Socioambiental Floranativa, atuou no Projeto de Desenvolvimento Sustentável (PDS) Terra Nossa, em Novo Progresso, Pará. Teve por objetivo principal reduzir a emissão e fixar o gás carbônico, por meio da introdução de práticas de restauração (implantação de SAF), utilizando como instrumentos a capacitação dos agricultores familiares e assentados da reforma agrária em ações voltadas a recuperação de ambientes degradados localizados em áreas de preservação permanente e reserva legal com ações de reflorestamento.

A implantação de SAFs é uma das opções entre os sistemas de produção, tendo o objetivo de contribuir para a segurança alimentar e o bem-estar social e econômico dos produtores rurais de baixa renda, assim como para a conservação dos recursos naturais (ARCO-VERDE, 2008). O agricultor tem inúmeras opções para compor um sistema agroflorestal ou mesmo para práticas simples, como uma cerca viva ou o sombreamento de uma pastagem, desde que ele conheça bem as plantas que escolhe, pois, o resultado ocorrerá a médio e longo prazo (MEIRELLES et al., 2003).

Já a análise de viabilidade econômica tem como objetivo examinar os custos e benefícios em função dos preços de mercado possibilitando refletir a provável viabilidade de um empreendimento ou projeto ao longo do tempo. $\mathrm{O}$ importante no momento de selecionar modelos agroflorestais viáveis economicamente é conhecer previamente à implantação, os custos de cada fase, a demanda de mão de obra e a rentabilidade do SAF podendo mensurar quando serão realizadas as atividades produtivas e o fluxo real de custos e ingressos durante o período da análise e o balanço final do investimento (ARCO-VERDE et al., 2014; ARCOVERDE, 2008).

Os estudos a respeito da viabilidade econômica de SAFs ocupam a terceira colocação em pesquisas no tema com 11,3\% das publicações entre os anos de 2005 a 2015 (SOUSA, 2016). A demanda por informações acerca da viabilidade econômica de sistemas agroflorestais tem crescido na medida em que os produtores buscam diversificação e aumento de renda. 
Diante disso, faz-se necessário o aprofundamento em pesquisas sobre o tema em SAFs possibilitando ao produtor a tomada de decisões em seu investimento antes mesmo destes serem implementados para minimizar frustrações e perda de capital. Nesse sentido, o objetivo deste trabalho foi realizar uma estimativa de geração de renda, por meio de uma análise de viabilidade econômica, a partir da implantação de sistemas agroflorestais por agricultores familiares, possibilitando ao produtor a tomada de decisões em seu investimento antes mesmo deste ser implementado para minimizar frustrações e perda de capital.

\section{MATERIAIS E MÉTODOS}

O trabalho foi realizado no Projeto de Desenvolvimento Sustentável (PDS) Terra Nossa, criado no ano de 2006 (Portaria/Incra N.3 de 06 de junho de 2006) localizado nos municípios de Novo Progresso e Altamira com acesso principal pela Rodovia BR-163 (figura 1). Este assentamento possuía inicialmente uma área de 149.842,4 ha que posteriormente foi alterada (retificação no Diário Oficial da União no dia 13 de março de 2015) para 20.081 ha com previsão de alocação de 373 unidades familiares beneficiárias (INCRA, 2015), sendo que, de acordo com informações coletadas junto a agentes locais, somente 161 famílias praticam alguma atividade agrícola ou possuem residência no assentamento.

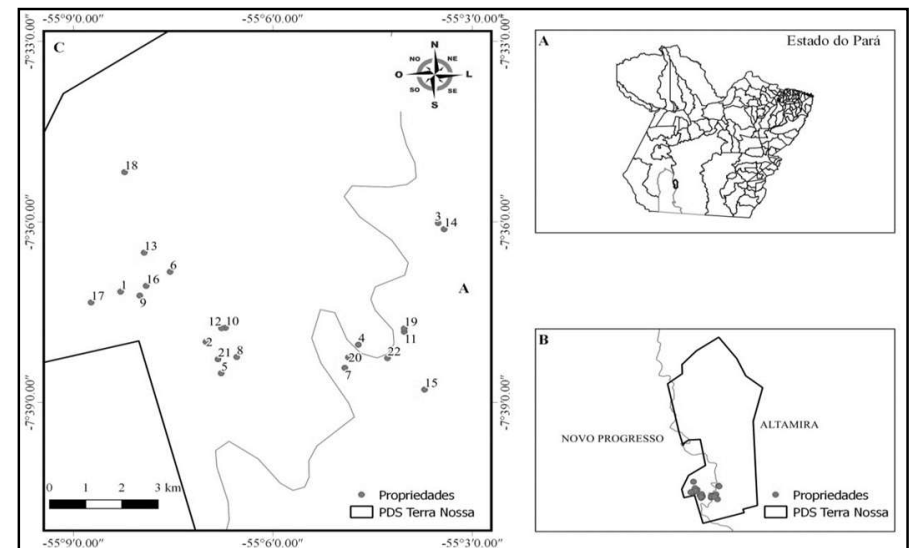

Figura 1: Localização das áreas de estudo - PDS Terra Nossa, Novo Progresso (PA).

Ao todo, 44 unidades familiares distribuídas aleatoriamente no assentamento demonstraram interesse em participar da implantação de SAF promovidos pelo Projeto Horizonte Verde. Para a coleta de dados deste trabalho foram selecionadas 18 propriedades nas quais os proprietários já haviam escolhido o que plantar, ou já estavam com mudas postas em campo. Aplicou-se questionário in loco em forma de entrevista pessoal. A aplicação do questionário visou prioritariamente obter informações sobre a escolha das espécies para a implantação de sistemas agroflorestais bem como a pretensa destinação dos produtos oriundos destes cultivos.

Para compor o valor das receitas, obtiveram-se dados sobre os preços de mercado dos produtos comercializados na sede municipal de Novo Progresso, por meio de aplicação de questionário semiestruturado, para fins de constatação dos valores de produtos como sementes, frutos, madeira, cereais entre outros produtos.

Na composição dos custos, foram coletados dados sobre o preparo e tamanho da área, custos de mão de obra, vegetação anterior ao plantio do SAF, espaçamento e adubação e procedência de mudas. 0 
custo de mão de obra (diária) e hora máquina, praticados no PDS Terra Nossa e utilizados neste estudo, são de $\mathrm{R} \$ 60,00$ e $\mathrm{R} \$ 125,00$, respectivamente. Para uma melhor representação dos custos também foram utilizados, como parâmetros gerais, dados de preparo e manutenção do SAF implantado na Fazenda da Universidade Federal do Oeste do Pará (UFOPA).

Quanto à manutenção dos sistemas, ao longo dos anos foram considerados dados de tratos culturais encontrados nos estudos de Rêgo (2014), em propriedades rurais em no município de Alenquer, e de Mendes (2003) em SAFs no município de Tomé-Açu, também no estado do Pará. O horizonte de planejamento e inferências econômicas do estudo correspondeu a 20 anos considerando o limite máximo de tempo para financiamentos agrícolas referentes às culturas de longos períodos de maturação adotado pelo Fundo Constitucional de Financiamento do Norte (LUCENA et al., 2016).

Os dados coletados foram processados por meio da planilha de cálculos de indicadores financeiros para sistemas agroflorestais desenvolvida pela Embrapa em 2014 denominada AmazonSAF (ARCO-VERDE, 2014). Através desta planilha calcularam-se os valores de Valor Presente Líquido (VPL), Taxa Interna de Retorno (TIR) e Relação Beneficio-Custo (RB/C) que são os principais índices utilizados como parâmetros econômicos para a análise financeira de SAFs (BENTES-GAMA, 2005; SANGUINO et al., 2007; ARCO-VERDE, 2008; PALHETA, 2014).

Segundo Santana (2005) para análise de viabilidade econômica de SAFs o Valor Presente Líquido (VPL) é considerado o indicador financeiro mais robusto, pois estima o valor a preço de hoje do fluxo de caixa, levando em conta uma determinada taxa de juros e quando o VPL for positivo considera-se o empreendimento viável. A Taxa Interna de Retorno (TIR) é a taxa de juros que iguala o valor presente dos benefícios ao valor presente de custos (ARCO-VERDE, 2014). O empreendimento é considerado viável se a TIR for maior que a Taxa Mínima de Atratividade (TMA). Já a Relação Benefício Custo (RB/C) indica o quanto os benefícios superam ou não os custos totais onde o projeto é viável se o valor for maior ou igual à unidade de capital (BÖRNER, 2009).

A taxa de juros utilizada para remunerar o capital investido nos projetos foi de $8,75 \%$ ao ano, que é a taxa de juros adotada no Plano Safra 2015/2016 estipulada pelo Banco Central do Brasil para linha de crédito na agricultura. Para a estimativa de geração de renda com produtos agroflorestais, foram utilizados dados de produção oriundos da literatura (tabela 1), adaptados para a densidade de plantio de cada SAF avaliado no estudo. Para o valor de renda utilizou-se o preço de mercado dos produtos para compra por feirantes ou intermediários constatados através da pesquisa realizada em Novo Progresso.

Tabela 1: Produtos agroflorestais e produtividade utilizada como parâmetro para produção das espécies presentes nos SAFs avaliados.

\begin{tabular}{|l|l|c|c|c|}
\hline Espécie & Unidade & Produtividade* & Anos & Fonte \\
\hline Açaí & $\mathrm{kg} / \mathrm{ha}$ & $1000-4500$ & 3o ao 20 & Oliveira, 2007 \\
\hline Andiroba & $\mathrm{kg} / \mathrm{ha}$ & 1890 & 5o & Ehrich,1999 \\
\hline Banana & $\mathrm{kg} / \mathrm{ha}$ & $165-285$ & 20 ao 60 & Arco-Verde, 2008 \\
\hline Cacau & $\mathrm{kg} / \mathrm{ha}$ & $285-1140$ & 3o ao 20 & Sanguino et al 2007 \\
\hline Castanheira & $\mathrm{kg} / \mathrm{ha}$ & $434-2294$ & 70 ao 20 & Arco-Verde, 2014 \\
\hline Cumaru & $\mathrm{kg} / \mathrm{ha}$ & $63-1063$ & 40 ao 20 & Rêgo, 2014 \\
\hline Cupuaçu & $\mathrm{kg} / \mathrm{ha}$ & $6-660$ & 5o ao 20 & Arco-Verde 2008 \\
\hline Cedro & $\mathrm{m}$ 3/ha & 65 & 200 ano & Campos-Filho, 2015 \\
\hline
\end{tabular}


SILVA, S. U. P.; PAULETTO, D.; MOTA, C. G.; NASCIMENTO, G. C. S.; SANTOS, J. A. C.; RODE, R.; NOCE, $R$.

\begin{tabular}{|l|l|c|c|c|}
\hline Copaíba & $\mathrm{I} / \mathrm{ha}$ & 1,3 & 10 o ao 20 & Pastore \& Leite, 2003 \\
\hline Ipê amarelo & $\mathrm{m}^{3} / \mathrm{ha}$ & 66 & 20 o ano & Campos-Filho, 2015 \\
\hline Mogno africano & $\mathrm{m}^{3} / \mathrm{ha}$ & 60 & 15 o ano & Sanguino et al 2007 \\
\hline Milho & $\mathrm{kg} / \mathrm{ha}$ & 2200 & 10 ao 3 ano & Perin, 2011 \\
\hline Pau de balsa & $\mathrm{m}^{3} / \mathrm{ha}$ & 180 & 10 e 3o anos & Reis, 2011 \\
\hline
\end{tabular}

Legenda: *Intervalo mínimo e máximo de produção em 20 anos. Observação: produtividade adaptada de acordo com a densidade de plantas do SAF do produtor.

Para uma estimativa mais precisa do cenário de renda nos SAFs acrescentou-se o percentual de $10 \%$ de perdas de mudas em campo. Para preservar a identidade do produtor e melhor identificação das propriedades, adotaram-se as siglas S1 até S18 para se referir aos arranjos implantados pelos agricultores. Em virtude da dificuldade de detalhar os 18 arranjos implantados pelos produtores optou-se por informar as espécies usadas nos SAFS, juntamente com os espaçamentos adotados e a densidade de plantas por hectare para facilitar o entendimento dos consórcios (tabela 2).

Tabela 2: Espécies implantadas, espaçamento adotados e densidade nos arranjos para 1 hectare de SAF em Novo Progresso.

\begin{tabular}{|c|c|c|c|c|c|c|c|c|c|c|}
\hline Nome popular & Nome científico & \multicolumn{9}{|c|}{ Espaçamento (m) } \\
\hline & & 0,5 & $2,5 \times 2,5$ & $3 \times 3$ & $4 \times 4$ & $5 \times 5$ & $9 \times 6$ & $9 \times 9$ & $12 \times 6$ & $10 \times 10 m^{*}$ \\
\hline Açaí & Euterpe oleracea Mart. & & & $\mathrm{X}$ & $\mathrm{X}$ & $\mathrm{x}$ & & $\mathrm{x}$ & & \\
\hline Andiroba & Carapa guianensis & & & & & & & $\mathrm{x}$ & & \\
\hline Banana & Musa sp. & & $\mathrm{x}$ & $x$ & $x$ & & & & & \\
\hline Cacau & Theobroma cacao L. & & & $\mathrm{X}$ & & & & & & \\
\hline Castanheira & Bertholletia excelsa H. B. K & & & & & & & $\mathrm{x}$ & & \\
\hline Cedro & Cedrela fissilis & & & & & & $\mathrm{x}$ & $x$ & & \\
\hline Copaíba & Copaifera $s p \mathrm{~L}$. & & & & & & & $x$ & $\mathrm{x}$ & \\
\hline Cumaru & Dipteryx odorata Willd & & & & & & $x$ & $x$ & $x$ & \\
\hline Cupuaçu & $\begin{array}{c}\text { Theobroma grandiflorum Willd.ex } \\
\text { Spreng. Schum }\end{array}$ & & & $x$ & $x$ & & & & & \\
\hline Ingá & Inga sp. & & & & & & & $\mathrm{x}$ & & $x$ \\
\hline Ipê & Handroantus serratifolia & & & & & & $\mathrm{x}$ & $\mathrm{x}$ & & \\
\hline Jatobá & Hymenaea coubaril & & & & & & $x$ & $x$ & & \\
\hline Milho & Zea mays & $x$ & & & & & & & & \\
\hline Mogno africano & Khaya Ivorensis A. Chevalier & & & & & & $\mathrm{x}$ & $\mathrm{x}$ & & \\
\hline Nim indiano & Azadirachta indica & & & & & & & $\mathrm{x}$ & & $x$ \\
\hline Pau de balsa & $\begin{array}{c}\text { Ochroma pyramidale (Cav. ex } \\
\text { Lamb.) Urban }\end{array}$ & & & & & & $x$ & $x$ & & \\
\hline Densidade (n/ha) & & 40000 & 1600 & 1112 & 625 & 400 & 185 & 123 & 138 & 40 \\
\hline
\end{tabular}

Legenda: *plantio realizado somente nas bordas do SAF.

\section{DISCUSSÃO TEÓRICA}

\section{Análise financeira}

Na tabela 3 são apresentados os indicadores econômicos em um horizonte de 20 anos para os SAFs implantados no PDS Terra Nossa. Utilizando-se a taxa de juros de $8,75 \%$ ao ano grande parte dos arranjos de SAFs analisados mostrou-se ter uma estimativa de renda positiva pois os valores de VPL foram positivos em um horizonte de 20 anos. Ao analisar a TIR, com exceção do arranjo S4, todos os arranjos obtiveram percentuais acima da TMA (8,75\%) podendo-se inferir que todos os demais arranjos são viáveis. Quanto a $\mathrm{RB} / \mathrm{C}$, percebe-se que todos os SAFs, com exceção do S4, proporcionam lucratividade ao produtor, pois apresentam retorno de capital maior do que uma unidade de real investida. Com exceção do arranjo S4, todos os SAFs atestaram valores de indicadores financeiros positivos. 
Tabela 3: Indicadores financeiros (VPL, TIR, RB/C), receitas e despesas em um horizonte de 20 anos para 1,0 hectare de SAF com diferentes espécies no PDS Terra Nossa.

\begin{tabular}{|c|c|c|c|c|c|c|}
\hline SAF & Espécies Componentes do SAF & Receita & Despesa & VPL & TIR & $\mathrm{RB} / \mathrm{C}$ \\
\hline S18 & $\mathrm{Aç}, \mathrm{Ca}, \mathrm{Cp}, \mathrm{Cu}, \mathrm{Pb}, \mathrm{Ne}{ }^{*}, \mathrm{Ce}, \mathrm{Co}, \mathrm{Mo}, \mathrm{Ip}$ & $396.469,30$ & $147.310,29$ & $249.159,01$ & $38,84 \%$ & 2,70 \\
\hline S2 & $\mathrm{Ba}, \mathrm{Aç}, \mathrm{Ca}, \mathrm{Cp}, \mathrm{Cu}, \mathrm{Mo}$ & $356.853,75$ & $145.710,32$ & $211.143,43$ & $37,61 \%$ & 2,40 \\
\hline S11 & $\mathrm{Aç}, \mathrm{Cu}, \mathrm{Ct}, \mathrm{Ce}, \mathrm{Mo}$ & $240.527,25$ & $98.500,11$ & $142.027,14$ & $31,39 \%$ & 2,40 \\
\hline S10 & $\mathrm{Cu}, \mathrm{Pb}, \mathrm{Ne}^{*}, \mathrm{Ce}, \mathrm{Mo}, \mathrm{Ip}, \mathrm{Ja}$ & $212.804,16$ & $64.835,24$ & $147.968,92$ & $29,60 \%$ & 3,30 \\
\hline S1 & $\mathrm{Mi}, \mathrm{Ba}, \mathrm{Aç}, \mathrm{Ca}, \mathrm{Cu}, \mathrm{Pb}, \mathrm{Ne}^{*}, \mathrm{Mo}$ & $251.392,84$ & $116.624,99$ & $134.767,84$ & $28,34 \%$ & 2,20 \\
\hline S17 & $\mathrm{Ba}, \mathrm{Aç}, \mathrm{Cp}, \mathrm{Cu}$ & $226.965,54$ & $114.030,67$ & $112.934,87$ & $27,60 \%$ & 2,00 \\
\hline S15 & $\mathrm{Ba}, \mathrm{Ca}, \mathrm{Cu}$ & $177.821,63$ & $78.544,24$ & $99.277,39$ & $27,06 \%$ & 2,30 \\
\hline S16 & $\mathrm{Cu}, \mathrm{Pb}, \mathrm{Ne}^{*}, \mathrm{Mo}, \mathrm{Co}$ & $173.244,10$ & $66.730,84$ & $106.513,26$ & $26,46 \%$ & 2,60 \\
\hline S5 & $\mathrm{Ca}, \mathrm{Cp}, \mathrm{Cu}, \mathrm{Ce}, \mathrm{Ip}, \mathrm{Ja} \mathrm{*}^{*}, \mathrm{Mo}$ & $203.972,61$ & $89.959,98$ & $114.012,64$ & $25,19 \%$ & 2,30 \\
\hline S7 & $\mathrm{Cp}, \mathrm{Cu}, \mathrm{Pb}, \mathrm{Ct}, \mathrm{Ne}^{*}, \mathrm{Ce}, \mathrm{Co}, \mathrm{Mo}$ & $188.402,04$ & $80.628,28$ & $107.773,76$ & $24,87 \%$ & 2,30 \\
\hline S13 & $\mathrm{An}, \mathrm{Cu}, \mathrm{Mo}$ & $149.912,49$ & $65.515,11$ & $84.397,38$ & $24,60 \%$ & 2,30 \\
\hline S12 & $\mathrm{Cp}, \mathrm{Ca}, \mathrm{Cu}, \mathrm{Ne}^{*}, \mathrm{Ce}, \mathrm{Co}, \mathrm{Mo}$ & $223.033,72$ & $10.231,33$ & $118.802,38$ & $24,60 \%$ & 2,10 \\
\hline S8 & $\mathrm{Cu}, \mathrm{Pb}, \mathrm{In} *, \mathrm{Mo}$ & $130.038,36$ & $62.316,74$ & $67.721,62$ & $22,01 \%$ & 2,10 \\
\hline S3 & $\mathrm{Cu}, \mathrm{Pb}, \mathrm{In} *, \mathrm{Mo}$ & $130.038,36$ & $63.728,97$ & $66.309,38$ & $21,84 \%$ & 2,00 \\
\hline S9 & $\mathrm{Ba}, \mathrm{Cp}, \mathrm{Cu}, \mathrm{Ct}, \mathrm{Ce}, \mathrm{Mo}$ & $171.404,30$ & $90.708,54$ & $80.695,76$ & $20,82 \%$ & 1,90 \\
\hline S6 & $\mathrm{Ba}, \mathrm{Aç}, \mathrm{Cu}, \mathrm{Pb}, \mathrm{In}^{*}, \mathrm{Ce}, \mathrm{Mo}, \mathrm{Ip}$ & $164.546,35$ & $86.505,97$ & $78.040,38$ & $20,37 \%$ & 1,90 \\
\hline S14 & $A c ̧, C p$ & $103.886,84$ & $87.151,85$ & $16.734,99$ & $14,97 \%$ & 1,20 \\
\hline S4 & $\mathrm{Ba}, \mathrm{Cp}, \mathrm{An}, \mathrm{Mo}$ & $36.751,59$ & $77.338,99$ & $-40.587,40$ & $x x x$ & 0,50 \\
\hline
\end{tabular}

Legenda: Aç: açaí; An: andiroba; Ba: banana; Ca: cacau; Ct: castanheira; Cu: cumaru; Cp: cupuaçu; In: ingá; Mi: milho; Ne: neem; Pb: pau de balsa; Mo: mogno africano; Ce: cedro; Ip: ipê; Co: copaíba; Ja: jatobá. *Espécies sem valor produtivo para análise econômica.

\section{Receitas futuras, saldos e despesas estimadas nos SAFs}

Em relação às receitas e saldos nos SAF observa-se que os arranjos mais rentáveis (> VPL) foram os arranjos S18, S2, S10, S11, S1 e S12 (tabela 3). A grande maioria dos arranjos possui em sua composição açaí e cumaru. Quanto às despesas, propostas na tabela 3, observam-se custos altos na análise dos SAFs para um horizonte de 20 anos. A figura 2 representa a previsão de receitas totais, custos e fluxo de caixa do SAF S18 por hectare que se apresentou como o mais rentável de acordo com critério do VPL, onde pode-se observar a proporção entre as receitas e os custos em cada ano do desenvolvimento do projeto. O SAF considerado o mais rentável (S18) apresentou uma demanda de mão de obra crescente a partir do segundo ano, por volta de 200 diárias/ha ano (figura 3).

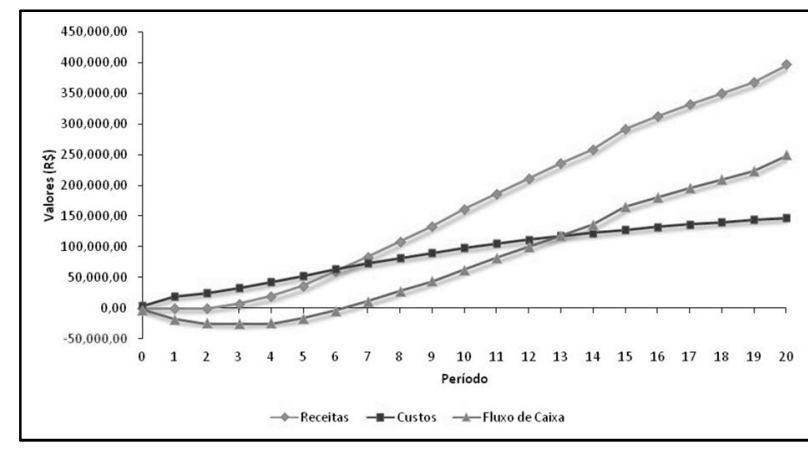

Figura 2: Previsão de receitas, custos totais e fluxo de caixa em um horizonte de 20 anos para o arranjo S18.

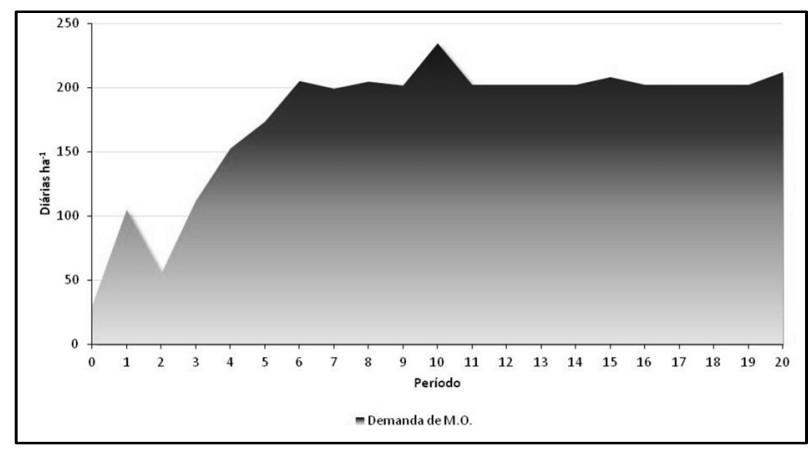

Figura 3: Demanda Total de Mão de Obra em um horizonte de 20 anos para o arranjo S18.

\section{DISCUSSÃO}

Os valores de VPL positivos para um horizonte de 20 anos (Tabela 3) encontrados no presente estudo concordam com experiências de SAFs na Amazônia que demonstraram ser economicamente viável a implantação deste tipo de cultivo (BENTES-GAMA, 2005; ARCO-VERDE et al., 2013; PALHETA, 2014). Os resultados da TIR acima da TMA (8,75\%) (tabela 3), com exceção do arranjo S4, apontam viabilidade dos SAFs, 
pois, conforme cita Arco-Verde et al. (2014), o empreendimento é considerado viável se a TIR for superior a TMA. Esses valores são superiores a resultados de Paula (2011) que avaliando a viabilidade econômica de um SAF em Santa Bárbara no Pará encontrou valor da TIR na ordem de 18,63\% com taxa de juros de 10\% ao ano.

Os valores de RB/C em todos os SAFs foram lucrativos, com exceção do arranjo S4 (tabela 3). Esses valores assemelham-se a estudos de viabilidade econômica de SAFs na Amazônia que provaram ser rentáveis (SANGUINO et al., 2007; PARAENSE, 2013; PALHETA, 2014). O provável insucesso do arranjo S4, composto por banana, cupuaçu, andiroba e mogno africano, está ligado com as opções restritas de mercado local para espécies escolhidas pelo produtor.

O preço da semente de andiroba na venda para extração de óleo no mercado local é baixo $(\mathrm{R} \$ 0,70 / \mathrm{kg})$. Já o mogno africano, com ênfase no produto madeireiro, somente poderá ser colhido aos 15 anos (SANGUINO et al., 2007) o que irá conferir um retorno tardio ao produtor. Além disso, a banana e o cupuaçu são espécies que requerem muitos cuidados com manutenção e tratos culturais o que onera os custos de produção. Todos esses fatores culminaram por onerar o arranjo S4, tornando-o inviável baseado nos indicadores financeiros deste estudo.

Arranjos mais rentáveis de acordo com critério de VPL, arranjos S18, S2, S10, S11, S1 e S12 (tabela 3), apresentaram maiores receitas e saldos. Valores esses semelhantes aos resultados encontrados por Aires (2003) numa projeção de renda de sistemas agroflorestais em assentamentos no estado de Rondônia. O fato da grande presença de açaí e cumaru nos SAFs também é demonstrado por Mendes (2003) que confirma o uso dessas espécies dentre as mais frequentes em SAFs nas propriedades rurais de Tomé-Açu, Pará. Essas espécies têm alta produtividade e retorno financeiro rápido. O açaí pode chegar ao terceiro ano com produtividade de 1.000 a $4.500 \mathrm{~kg} / \mathrm{ha}$ de fruto por hectare (OLIVEIRA, 2007) e o cumaru no quarto ano com produção de 63 a $1.063 \mathrm{~kg} / \mathrm{ha}$ (RÊGO, 2014).

As espécies na sucessão ecológica do tipo clímax tiveram uma notável importância na composição das receitas futuras nos arranjos estudados, quando considerado sua renda em longo prazo, pois poderão fornecer diversidade de produtos como óleos, sementes e madeira. Nessa categoria destacam-se as seguintes espécies as escolhidas pelos produtores: mogno africano, cedro, cumaru, copaíba, pau de balsa, andiroba e ipê. Lucena et al. (2016), estudando a viabilidade econômica de um modelo agroflorestal em Altamira, Pará verificou desempenho satisfatório de espécies florestais à medida que as receitas provenientes da madeira e sementes foram sendo incorporadas a rentabilidade do sistema.

Os SAFs apresentaram despesas altas para uma análise de 20 anos (tabela 3). Bentes-Gama (2003) constatou que SAFs apresentam custos superiores se comparados a monocultivos onde destaca que apesar dos elevados custos eles geram receitas altas por hectare. Verificou-se que os maiores custos nos três primeiros anos são com preparo de área e insumo. Palheta (2014) destaca a amortização dos custos de implantação com introdução de cultivos anuais, uma vez que os custos com espécies de ciclo longo dificultam o rápido retorno do projeto. Nos anos seguintes as despesas são compostas por manutenção nos plantios com tratos silviculturais como poda e colheita. Percebe-se que os arranjos mais rentáveis incluem uma ou mais espécies que demandam esse tipo de cuidado, sendo elas: açaí, banana, cacau, cupuaçu e cumaru. 
Os tratos silvoculturais variam de acordo com as espécies que foram usadas nos arranjos, tendo em vista que algumas requerem alguns cuidados a mais do que as outras para se obter uma boa produtividade e consequentemente maior retorno financeiro. Os resultados corroboram com os estudos de Bentes-Gama (2005), em SAFs na Amazônia Ocidental em Rondônia, onde os tratos culturais e colheita representaram mais de $70 \%$ da composição dos custos totais.

No SAF considerado o mais rentável (figura 2), destaca-se que as receitas superam os custos a partir do sétimo ano, com tendência positiva até o último ano de avaliação do projeto. Esse comportamento pode ser visto nos estudos de Arco-Verde et al. (2013) que revelou receitas superiores aos custos a partir do oitavo ano.

Segundo Arco-Verde (2008) os maiores custos ocorrem nos três primeiros anos de implantação de um SAF, pois a fase de implantação é a mais onerosa e deve ser feita de forma que se evite maior concentração de despesas em um só ano. No entanto, o SAF considerado o mais rentável não seguiu essa linha de raciocínio, pois se observa que a partir do segundo ano tem-se uma demanda de mão de obra crescente, por volta de 200 diárias/ha ano (figura 3). Esse comportamento está ligado ao fato de que o SAF teve em sua composição dez espécies, sendo elas: açaí, cacau, cupuaçu, cumaru, pau de balsa, nim, cedro, copaíba, mogno africano e ipê. Entre estes componentes quatro deles (açaí, cacau, cupuaçu e cumaru) demandam quantidades maiores de diárias nos tratos culturais com poda e colheita.

O aumento de mão de obra no décimo ano no SAF apontado como mais rentável pela análise (figura 3), está relacionado à colheita do óleo de copaíba, por volta de 20 diárias/ha ano. Nota-se também um leve aumento da demanda de mão de obra nos anos 15 e 20. De acordo com Arco-Verde et al. (2013) esse fato está relacionado à colheita dos componentes madeiráveis, uma vez que nesses respectivos anos há necessidade de desbaste das espécies florestais com valor madeireiro.

\section{CONCLUSÕES}

De acordo com os indicadores de VPL, TIR e RB/C, grande parte dos SAFs analisados atestaram boa estimativa de renda, apontando viabilidade econômica, possibilitando assim ao produtor tomar decisões de investimento. O Arranjo S4 demonstrou ser inviável economicamente. Este fato constata que a viabilidade ou não de um projeto está ligada a tomada de decisões pelo produtor, sobretudo na escolha de espécies para compor os arranjos e as opções de mercado para os produtos. Espécies que requerem maiores gastos com tratos culturais, preço não competitivo no mercado e retorno tardio, são fatores que se combinados, acabam por desfavorecer a viabilidade do empreendimento.

Os SAFs mais rentáveis economicamente apresentaram em sua composição o açaí e cumaru, espécies essas com produtividade alta e valor atrativo no mercado local. Espécies florestais proporcionam retorno em longo prazo, sendo uma ótima opção quando se pensa em retornos de capital futuro. No entanto, percebese que nas escolhas dos arranjos os produtores não privilegiaram o uso de espécies anuais o que minimizaria os custos iniciais e proporcionaria retorno nos primeiros anos de projeto. 


\section{REFERÊNCIAS}

AIRES, K. S.. Estudo da viabilidade econômico-financeira de dois modelos de consórcios agroflorestais: Cacau (Theobroma cacao L.) x Café (Caffea arabica) x Teca (Tectoma Grandis) e Cacau (Theobroma cacao L.) x Pupunha (Bractis gasipaes) x Freijó-Louro (Cordia alliodora). Monografia (Graduação em Administração) - Universidade Luterana do Brasil, Ji-Paraná, 2003.

ARCO-VERDE, M. F.. Análise financeira de sistemas produtivos integrados. Colombo: Embrapa Florestas, 2014.

ARCO-VERDE, M. F.. Sustentabilidade biofísica e socioeconômica de sistemas agroflorestais na Amazônia brasileira. Tese (Doutorado em Ciências Florestais) Universidade Federal do Paraná, Curitiba, 2008.

ARCO-VERDE, M. F.; AMARO, G. C.. Cálculo de indicadores financeiros para sistemas agroflorestais. Boa Vista: Embrapa, 2014.

ARCO-VERDE, M. F.; AMARO, G. C.; SILVA, I. C.. Sistemas agroflorestais: conciliando a conservação do ambiente e a geração de renda nas propriedades rurais. Brasília: Embrapa, 2013.

AZEVEDO, A.; ALENCAR, A.; MOUTINHO, P.; RIBEIRO, V.; REIS, T.; STABILE, M.; GUIMARÃES, A.. Panorama sobre o desmatamento na Amazônia em 2016. Brasília: IPAM, 2016.

BENTES-GAMA, M. M.. Análise econômica de sistemas agroflorestais na Amazônia Ocidental, Machadinho D'OesteRO. Árvore, Viçosa, v.29, n.3, p.401-411, 2005.

BENTES-GAMA, M. M.. Análise técnica e econômica de sistemas agroflorestais 2003 em Machadinho d'Oeste, Rondônia. Tese (Doutorado em Ciências Florestais) Universidade Federal de Viçosa, Viçosa, 2003.

BÖRNER, J.. Serviços ambientais e adoção de sistemas agroflorestais na Amazônia: elementos metodológicos para análises econômicas integradas. In: PORRO, R.. Alternativa agroflorestal na Amazônia em transformação. Brasília: Embrapa, 2009.

CAMPOS-FILHO, E. M.. Guia de árvores com valor econômico. São Paulo: Agroícone, 2015.

EHRICH, C.. Visão das características agronômicas, operacionais e econômicas de espécies potencialmente agroflorestais no Acre. In: ENCONTRO DE FRUTAS NATIVAS DO NORTE E NORDESTE DO BRASIL. Anais. São Luís: Embrapa, 1999.

INCRA. Instituto Nacional de Colonização e Reforma Agrária. Ofício Incra/SR-30/G/n.428/2015. Santarém: INCRA, 2015.

INPE. Instituto Nacional de Pesquisas Espaciais. Monitoramento da Floresta Amazônica Brasileira por Satélite. São José dos Campos: INPE, 2007.
LUCENA, H. D.; PARAENSE, V. C.; MANCEBO, C. H. A.. Viabilidade econômica de um sistema agroflorestal com cacau e essências florestais de alto valor comercial em Altamira-PA. Revista de Administração e Negócios da Amazônia, v.8. n.1, 2016.

MEIRELLES, A. C. B.; MOTTER, C.; BELLÉ, N.. Revista dos Sistemas Agroflorestais. Alcântara: 2003.

MENDES, F. A. T.. Avaliação de modelos simulados de sistemas agroflorestais em pequenas propriedades cacaueiras selecionadas no município de Tomé-Açu, no estado do Pará. Belém: 2003.

OLIVEIRA, M. P.. Açaí: técnicas de cultivo e processamento. Fortaleza: Instituto Frutal, 2007.

PALHETA, I. C.; GOMES, C. A. S.; LOBATO, G. J. M.; PAULA, M. T.; PONTES, A. N.. Viabilidade econômica de um sistema agroflorestal no município de Santa Bábara-PA. Revista do Centro Científico Conhecer, Goiânia, v.10, n.19, p.1947, 2014.

PARAENSE, V. C.; MENDES, F. A. T.; FREITAS, A. D. D.. Avaliação Econômica de sistemas agroflorestais de cacau e mogno na transamazônica: um estudo de caso. Revista do Centro Científico Conhecer, Goiânia, v.9, n.16, p.2754, 2013.

PASTORE, F. J.; LEITE, F. H. F.. Óleo de copaíba na Amazônia: estudo de caso e potencial de produção. Brasília: 2003. PAULA, M. T.. Análise econômica de investimento de um sistema agroflorestal (SAF) no município de Santa Bárbara (PA). Curitiba: 2011.

PERIN, R.; SOUSA, J. N.; MORAES, R. R.; TONATO, F.; ROCHA, R. N. C.; FONTES, J. R. A.. Efeito do sombreamento na produtividade de milho em sistemas agrossilvopastoris. Manaus: Embrapa, 2011.

RÊGO, L. J. S.. Análise econômica da produção da amêndoa de cumaru e caracterização do seu mercado em Santarém e Alenquer, Pará. Dissertação (Mestrado) - Universidade Federal de Viçosa, Viçosa, 2014

REIS, C. A. F.. Estado da arte de plantios com espécies florestais de interesse para o Mato Grosso. Brasília: Embrapa, 2011.

SANGUINO, A. C.. Avaliação econômica de sistemas agroflorestais no Pará. Revista Ciências Agrárias, Belém, n.47, p.71-88, 2007.

SANTANA, A. C.. Elementos de economia, agronegócio e desenvolvimento local. Belém: UFRA, 2005

SOUSA, W. A.. Sistemas Agroflorestais: uma análise bibliométrica da produção científica de revistas brasileiras de 2005 a 2015. Monografia (Graduação em Engenharia Florestal) - Universidade Federal do Oeste do Pará, Santarém, 2016.

A CBPC - Companhia Brasileira de Produção Científica (CNPJ: 11.221.422/0001-03) detém os direitos materiais desta publicação. Os direitos referem-se à publicação do trabalho em qualquer parte do mundo, incluindo os direitos às renovações, expansões e disseminações da contribuição, bem como outros direitos subsidiários. Todos os trabalhos publicados eletronicamente poderão posteriormente ser publicados em coletâneas impressas sob coordenação da Sustenere Publishing, da Companhia Brasileira de Produção Científica e seus parceiros autorizados. Os (as) autores (as) preservam os direitos autorais, mas não têm permissão para a publicação da contribuição em outro meio, impresso ou digital, em português ou em tradução. 